\title{
Chronic Administration of the Resveratrol or N-PEP-12 Ameliorates the Endothelial Dysfunction in Aging Rats
}

\author{
Gonzalo Flores $^{1 *}$, Josue Hernández-Cabrera ${ }^{2}$, Celeste Santamaria-Juarez ${ }^{2}$, \\ Rubén Antonio Vázquez-Roque ${ }^{1}$, Elizabeth Monserrat-Hernández ${ }^{1}$, Ma. De Jesús Gómez-Villalobos ${ }^{1}$, \\ Jorge Flores-Hernández ${ }^{1}$, Fausto Atonal-Flores ${ }^{3}$, José Gustavo López-López ${ }^{2}$
}

\footnotetext{
${ }^{1}$ Instituto de Fisiología, Universidad Autónoma de Puebla, Puebla, México; ${ }^{2}$ Facultad de Ciencias Químicas, Universidad Autónoma de Puebla, Puebla, México; ${ }^{3}$ Facultad de Medicina, Universidad Autónoma de Puebla, Puebla, México.

Email: "gonzalo.flores@correo.buap.mx, "gonzaloflores56@gmail.com
}

Received November $27^{\text {th }}, 2013$; revised December $28^{\text {th }}$, 2013; accepted January $8^{\text {th }}, 2014$

Copyright (c) 2014 Gonzalo Flores et al. This is an open access article distributed under the Creative Commons Attribution License, which permits unrestricted use, distribution, and reproduction in any medium, provided the original work is properly cited. In accordance of the Creative Commons Attribution License all Copyrights @ 2014 are reserved for SCIRP and the owner of the intellectual property Gonzalo Flores et al. All Copyright (C) 2014 are guarded by law and by SCIRP as a guardian.

\begin{abstract}
Here we investigated the effects of chronic treated with resveratrol, a polyphenol (3,4,5-trihydroxystilbene) found in more than 70 plant species and food products such as red grapes, berries and peanuts, or N-PEP-12, a mix of peptides, on arteries in aging rats. Aging rats have shown endothelial dysfunction. Aged Sprague-Dawley rats $(18$ months old) were treated with resveratrol $(20 \mathrm{mg} / \mathrm{kg} / \mathrm{day}$ by gavage) or $\mathrm{N}-\mathrm{PEP}-12(60 \mathrm{mg} / \mathrm{kg} / \mathrm{day}$ by gavage) for 10 weeks. 24 hours after the last administration of resveratrol or N-PEP-12, the animals were weighed and diastolic and systolic blood pressure was measured by tail-cuff plethysmography. Thoracic and abdominal aorta artery segments were obtained from resveratrol-, N-PEP-12- and vehicle-treated rats and were mounted in isometric tension for isolated vessels. We found that resveratrol and N-PEP-12 improved acetylcholine-induced relaxation in the old animals. These results suggest that chronic administration of resveratrol or N-PEP-12 may in part reduce endothelial dysfunction in the aging.
\end{abstract}

\section{KEYWORDS}

Resveratrol; N-PEP-12, Aging; Vascular Endothelium; Blood Pressure

\section{Introduction}

Resveratrol (trans-3,4',5-trihydroxystilbene) is a nonflavonoid, polyphenolic compound found in various fruits and vegetables, and is particularly abundant in red grapes and red wine [1]. Additionally, the root extract of the weed Polygonumcuspidatum, an important constituent of Chinese and Japanese folk medicines, is a source of resveratrol [1]. Resveratrol has recently received attention for its benefit effects in neurodegenerative diseases, longevity and cardiovascular disease [1]. Cardiovascular diseases are among the most common cause of death among elderly patients in the world [2]. Age-specific mortality rates from heart disease and stroke increase exponentially with age throughout the later years of life, accounting for more than $40 \%$ of all deaths among

"Corresponding author. people aged 65 - 74 years and almost $60 \%$ at age 85 years and older [2]. Indeed, the effect of aging on the vascular system is considered a potential mechanism involved in vascular dysfunction and age-related atherosclerosis [2]. Interestingly, recent reports in rodents [3,4] and humans [5] suggest that increased activity of $\mathrm{NAD}(\mathrm{P}) \mathrm{H}$ oxidases leads to endothelial dysfunction in the aged [2] and that oxidative stress promotes the development of coronary artery disease and stroke in elderly patients $[2,4]$. Recently it has been showed resveratrol regulates NO levels in vascular endothelium [6]. Therefore, it is possible that the cardioprotective actions of resveratrol may function via an effect on endothelial dysfunction in the aging.

$\mathrm{N}-\mathrm{PEP}-12$ is a dietary supplement derived from Cerebrolysin and consists of neuropeptides and amino acids [7]. N-PEP-12 has been shown in vivo to enhance cogni- 
tive function and reduce neurodegenerative events associated with aging (Windisch et al., 2005). In healthy older adults, N-PEP-12 has been demonstrated to improve memory performance [7-9]. Resveratrol has also been shown to exert neuroprotective effects and has been indicated for the prevention and treatment of both acute and chronic neurodegenerative diseases, such as cerebral ischemia and Alzheimer's disease. In the present study we examined whether chronic administration of resveratrol or N-PEP-12 affects endothelial dysfunction during aging as evidenced by endothelia of the thoracic and abdominal aorta and blood pressure and cardiac rate in aged rats.

\section{Material and Methods}

\subsection{Animals}

A total of 40 Sprague-Dawley male rats, 14 months of age (weighing $573 \pm 24 \mathrm{~g}$ ) were obtained from our facilities from Claude Bernard bioterium (University of Puebla). Rats were housed in a temperature $\left(20^{\circ} \mathrm{C}-23^{\circ} \mathrm{C}\right)$ and humidity $(40 \%-50 \%)$ controlled environment on a 12 $12 \mathrm{~h}$ light-dark cycle with free access to food and water. All experimental procedures were approved by the IF-BUAP Animal Care Committee and are in accordance with the technical guidelines for production, care, and use of animals in the laboratory issued by SAGARPA México (NOM-062 ZOO-1999) and by the National Institutes of Health Guide for the Care and Use of Laboratory Animals. All efforts were made to minimize animal suffering and to reduce the number of animals used.

\subsection{Resveratrol or N-PEP-12 Administration}

In order to assess possible beneficial effects of resveratrol or N-PEP-12 on functional endothelial changes that occur in the vasculature during aging, rats were then administrated with resveratrol $(20 \mathrm{mg} / \mathrm{kg}$ by gavage; from Sigma-Aldrich, St. Louis, MO, USA) or N-PEP-12 (60 $\mathrm{mg} / \mathrm{kg}$ by gavage; from Ever NeuroPharma GmbH, Unterach, Austria) or an equal volume of vehicle was administrated every day in the morning (10:00 to 12:00 am) for a period of 10 weeks.

\subsection{Measure of Blood Pressure}

Blood pressure was measured in all the animals ( $n=9$ 11 per group). Systolic and diastolic blood pressure were measured using the tail-cuff method (XBP1000 Rat tail, Blood Pressure system, Kent Scientific Corporation). Systolic and diastolic blood pressures (mean \pm SE, mm $\mathrm{Hg}$ ) for the resveratrol-treatment, N-PEP-12-treated and vehicle-treated rats at 18 months old measured as previously described [10]. Blood pressure and heart rate of the vehicle, resveratrol- and N-PEP-12- treated rats were measured on day after of the last doses of resveratrol, N-PEP-12 or vehicle.

\subsection{Vascular Contractility}

Immediately after measuring the heart rate and blood pressure, animals ( $\mathrm{n}=9-11$ per groups) were deeply anesthetized with sodium pentobarbital $(75 \mathrm{mg} / \mathrm{kg}$ body weight, ip) and sacrificed. In addition 8 vehicle-treated adult Sprague-Dawley rats (3 months old and weighing between 250 to $300 \mathrm{~g}$ ) were also sacrificed as controls. Descending thoracic aorta were rapidly isolated and cleaned from perivascular tissue as described [11]. Briefly, the aortas were placed in Krebs' solution of the following composition: $\mathrm{NaCl} 133 \mathrm{mM}, \mathrm{KCl} 4.7 \mathrm{mM}$, $\mathrm{NaH}_{2} \mathrm{PO}_{4} 1.35 \mathrm{mM}, \mathrm{NaHCO}_{3} 16.3 \mathrm{mM}, \mathrm{MgSO}_{4} 0.61$ $\mathrm{mM}$, D-glucose $7.8 \mathrm{mM}$, and $\mathrm{CaCl}_{2} 2.52 \mathrm{mM}$ at $\mathrm{pH}$ 7.4. The aortas were cut into rings ( 2 - $3 \mathrm{~mm}$ in length). The rings were suspended horizontally by means of two parallel L-shaped stainless steel holders inserted into the lumen in 5-ml organ baths filled with Krebs' solution, bubbled with a $95 \% \mathrm{O}_{2}-5 \% \mathrm{CO}_{2}$ gas mixture, and maintained at $37^{\circ} \mathrm{C}$. One holder served as an anchor and the other was attached to an isometric force-displacement transducer coupled to Powerlab data acquisition and analysis system (AD Instruments). Contractile tension was recorded using the chart 5.0 computer program as previously described [11]. Each ring was stretched to a resting tension of $2 \mathrm{~g}$ (thoracic aorta) and allowed to equilibrate for 60 to $90 \mathrm{~min}$. During this period, tissues were re-stretched and washed every 30 min with warm Krebs' solution. After equilibration, rings with endothelium-intact were precontracted using $10^{-6} \mathrm{M}$ phenylephrine, and concentration-response curves to acetylcholine $\left(10^{-9}\right.$ to $\left.10^{-5} \mathrm{M}\right)$ were performed by cumulative addition. To avoid interferences with endogenous NO, the endothelium was removed in some preparations by gently rubbing the intimal surface of the rings with a metal rod. The endothelium removal procedure was verified by the inability of acetylcholine $\left(10^{-6} \mathrm{M}\right)$ to relax arteries precontracted with norepinephrine $\left(10^{-6} \mathrm{M}\right)$. In rings without endothelium, after equilibration, rings were precontracted by $80 \mathrm{mM} \mathrm{KCl}$, and, once a stable contraction was reached, were washed with warm Krebs solution for 30 minutes and concentration-response curves to phenylephrine $\left(10^{-9}\right.$ to $\left.10^{-5} \mathrm{M}\right)$ were performed by cumulative addition.

\subsection{Statistical Analysis}

Data on vascular contractility were analyzed by two-way ANOVA, followed by the Newman-Keuls test for posthoc comparisons, with age and drug as independent factors ( $P<0.05$ was considered significant). Whereas data from blood pressure test were analyzed by Mann-Whit- 
ney and Kruskal Wallis tests $(P<0.05$ considered significant).

\section{Results}

\subsection{Blood Pressure and Cardiac Rate}

Blood pressure and cardiac rate of the vehicle-, N-PEP12- and resveratrol-treated 18-month old rats were measured on the day after the last dose (Table 1). Systolic and diastolic blood pressure and the cardiac rate were also measured. Kruskal Wallis test statistical analysis revealed no significant differences between vehicle-, NPEP12- or resveratrol-treated rats (Table 1).

\subsection{Body Weight}

Bodyweight of the vehicle-, N-PEP-12- and resveratroltreated rats, were measured both before the first dose of resveratrol or N-PEP-12 and then again one day after the last dose (Table 1). Ten weeks of resveratrol or NPEP-12 administration did not alter body weight when compared to the vehicle-treated animals (By two-way ANOVA analysis, age; $F_{1,56}=6.18, P=0.01$; drugs; $F_{2,56}$ $=0.1, P=0.9$ and an interaction of drugs with age $F_{2,56}=$ $0.2, P=0.8$ ) (Table 1 ).

\subsection{Resveratrol and N-PEP-12 Improved the Vasodilation in the Old Arteries}

In rat aortic rings aged (without endothelium), phenylepherine induced a sustained vasoconstriction in a dosedependent manner in the isolated aortain all experimental groups (Figure 1(a)). Whereas acetylcholine induced a concentration-dependent relaxation in all vessels precontracted by phenylephrine (Figure 1(b)). A two-way ANOVA of phenylephrine-induced vasoconstriction (Figure 1(b)) revealed significant main effects of treatment $\left(F_{3,273}=6.3, P<0.01\right)$, doses $\left(F_{9,273}=369, P<\right.$ $0.01)$, and treatment by doses interaction $\left(F_{27,273}=1.8, P\right.$ $=0.01$ ); however, post hoc test revealed that ten weeks of resveratrol or N-PEP-12 administration did not modify the response to phenylephrine compared to the vehicletreated animals. Interestingly, the response to acetylcholine was modified in the vessels of the resveratrol- and NPEP-12-treated rats (by two-way ANOVA analysis, treatment; $F_{3,263}=5.5, P<0.01$; doses; $F_{9,263}=339, P<$ 0.9 and an interaction of treatment with doses $F_{27,263}=$ 1.56, $P=0.04$ ) (Figure 1(b)). Resveratrol and NPEP-12 adminstration improved the acetylcholine-induced relaxation in the old animals. Finally, vessels of the vehicletreated old rats exhibited a reduction inphenylephrineinduced contraction and acetylcholine-induced relaxation, compared to the vessels of the adult animals.

\section{Discussion}

The major aim of the present study was to investigate the effects of 10 weeks of resveratrol or N-PEP-12 administration on vasoconstriction and relaxation of the thoracic aorta of 18 months old rats. Interestingly, resveratrol, a polyphenol and N-PEP-12, a mix of peptides, both caused changes in the relaxation of the aorta of the aging rats. Aorta from resveratrol- or N-PEP-12-treated rats displayed enhanced relaxation produced by acetylcholine. The results from this study are consistent with a recent report [12], which demonstrated that aortae from old WistarKioto rats (20 - 24 months old) incubated with resveratrol $(10 \mu \mathrm{mol} / \mathrm{L})$ by one hour improved the acetylcholine-induced relaxation. Therefore, the authors suggest that resveratrol may inhibit the endothelial dysfunction by aging. It is noteworthy to mention that in our experimental design the effects of resveratrol are the results of long-term changes in the dysfunctional endothelium in the aged rats. This approached is similar to use of the resveratrol in humans. In contrast to other experiments, in this study resveratrol or N-PEP-12 administration was stopped 24 hours before the experimental measurements were recorded, thus, our data further suggest that chronic resveratrol treatment in aged rats, without high blood pressure, did not modify the blood pressure and heart rate, but was able to restore the vasorelaxive abilities of the

Table 1. Effect of the N-PEP-12 and resveratrol on the blood pressure of old rats.

\begin{tabular}{cccc}
\hline & Vehicle-treated & N-PEP-12-treated & Resveratrol-treated \\
\hline Weight at the beginning & $564 \pm 20 \mathrm{~g}$ & $577 \pm 19 \mathrm{~g}$ & $573 \pm 24 \mathrm{~g}$ \\
Weight at the end & $629 \pm 26 \mathrm{~g}$ & $613 \pm 32 \mathrm{~g}$ & $620 \pm 20 \mathrm{~g}$ \\
Systolic blood pressures & $150.8 \pm 2.6 \mathrm{mmHg}$ & $142 \pm 4.1 \mathrm{mmHg}$ & $146.3 \pm 4.7 \mathrm{mmHg}$ \\
Diastolic blood pressures & $97.3 \pm 2.1 \mathrm{mmHg}$ & $93 \pm 2.8 \mathrm{mmHg}$ & $96.2 \pm 4.7 \mathrm{mmHg}$ \\
Heart rate & $369 \pm 14.2 \mathrm{bpm}$ & $418 \pm 19 \mathrm{bpm}$ & $392 \pm 11 \mathrm{bpm}$ \\
Heart weight & $1.62 \pm 0.07 \mathrm{~g}$ & $1.62 \pm 0.06 \mathrm{~g}$ & $1.64 \pm 0.06 \mathrm{~g}$ \\
\hline
\end{tabular}

Mean and standard error (SEM) relative to blood pressure and weight of the animals before and after N-PEP-12- or resveratrol-treated on old rats. Interestingly no differences were found between groups. 


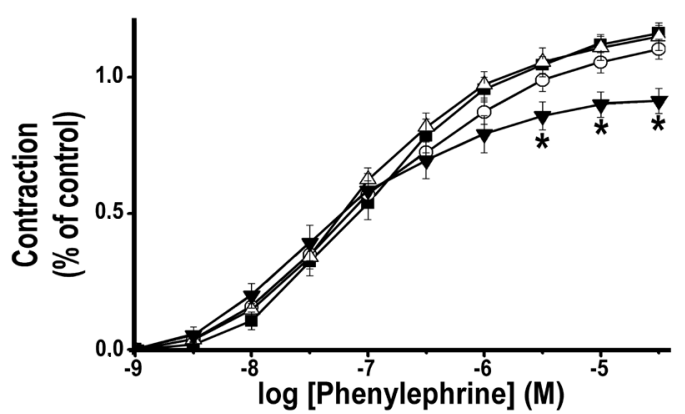

(a)

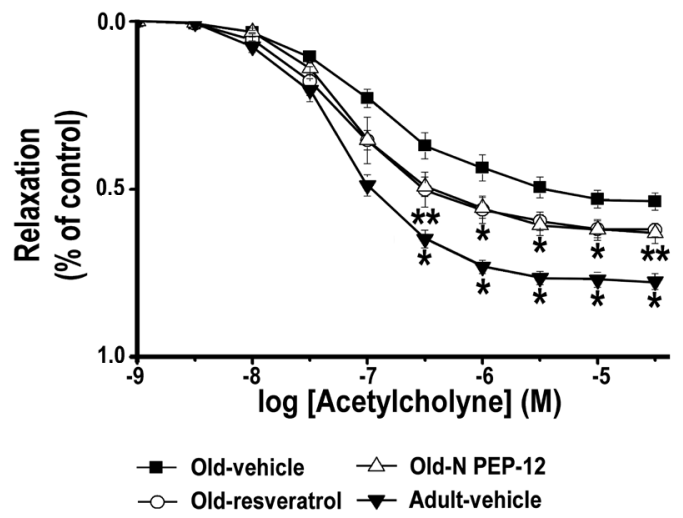

(b)

Figure 1. Effect of resveratrol or N-PEP-12 administration on vasoconstriction and relaxation of the isolated aortic rings from old animals. Values are means \pm S.E.M. $(n=8$ 11). ${ }^{*} \boldsymbol{P}<0.01$ versus old-vehicle; ${ }^{* *} P<0.05$ versus old-vehicle; ${ }^{+} \mathbf{P}<0.01$ versus adult-vehicle; ${ }^{++} \mathbf{P}<0.05$ versus adult-vehicle. (a) Concentration-response curve for contraction induced by phenylephrine. (b) Concentration-response curve for relaxation induced by acetylcholine.

aortae. It is noteworthy to mention that there is very scarce literature on N-PEP-12 and its effects on memory processes [7-9]. However, N-PEP-12 is derived from Cerebrolys in [7] which has been shown to have an antioxidant effects on the neurons [13].

In accordance with our results, a recent report suggests that chronic adminstration of resveratrol (10 to 20 weeks) did not modify the high systolic blood pressure of the adult spontaneously hypertensive rat (SHR) [14]. However, resveratrol increased compliance of small arteries of the SHR [14]. This effect may be due in part to a change in wall component stiffness. Whereas, in arteries of the young-adult WistarKioto rats, resveratrol reduced wall component stiffness, which explains the ability of resveratrol to increase compliance [14]. The authors suggest that a possible explanation could be related to the ability of resveratrol to limit the increase in compliance of SHR arteries, likely related to inhibitory effects on remodeling and the activation of the kinase-G that inhibited signal-regulated kinases (ERK)-enzymes rather than blood pressure or arterial wall component stiffness.
In addition, a recent in vitro report suggests that resveratrol may act through a novel cellular pathway that involves rapid stimulation of kinase-G system to inhibit the ERK1/2 mitogenic enzymes [15]. Interestingly these mechanisms remain functional in the absence of vascular endothelium [15], which implies that resveratrol may have effects even in advanced cases with endothelial damage. It should be noted that two recent reports have suggested that resveratrol inhibits blood pressure [16,17], These apparent differences from our findings are most likely due to methodological differences. The Ma et al., [16] study reports a microinjection of resveratrol into the rostral ventrolateral medulla (RVLM) dose dependently decreased blood pressure, heart rate and renal sympathetic nerve activity [16]. Whereas, in the other report, adult female rats were exposed to low levels of estradiaol-17 $\beta$ (E2) for 3 months which increases superoxide levels in RVLM and results in hypertension [17]. Interestingly, treatment with resveratrol reversed E2-induced increases in superoxide levels in the RVLM and reversed the increase in blood pressure [17]. It is generally accepted that RVLM plays a pivotal role in the regulation of vascular tone and the maintenance of blood pressure [18].

The effects of aging on the vascular system are complex, however the majority of the reports focus on vascular dysfunction and atherosclerosis. In vascular dysfunction several mechanisms have been proposed. It is well known that NO plays a crucial factor in the function of endothelial cells. One of the consequences of increased oxidative stress in aging is a functional inactivation of $\mathrm{NO}$ by high concentrations of $\mathrm{O}_{2}$-resulting in significant vasomotor dysfunction [2]. In opposition with the NO vascular dysfunction in aging, regular exercise improve endothelial function, possibly by augmenting NO bioavailability and attenuating oxidative stress [2]. Other beneficial effects of the exercise increased insulin sensitivity, decreased fat content, attenuation of hyperlipidemia and confers anti-inflammatory actions [2].

Several reports suggest that the effects of resveratrol may be mediated by NO synthesis and a decrease in $\mathrm{Ca}^{2+}$ influx [19]. However, a recent report suggests that resveratrol improved NO-mediated vasorelaxation without altering eNOS expression [20], possibly by virtue of its antioxidant actions. Therefore, resveratrol prevents free radical-induced degradation of $\mathrm{NO}$, thereby rescuing or increasing NO-cGMPPKG signaling. In addition, resveratrol-amplified phosphorylation of VASP at serine 239, a reliable marker of PKG activity [14]. As we mentioned before, cGMP-PKG signaling has been linked to suppression of ERK [15]; inhibition of ERK by resveratrol might thus be conferred by antioxidant-dependent augmentation of NO-cGMP-PKG signaling.

In summary, our data confirm and extend earlier stu- 
dies that in aging arteries with endothelial dysfunction, chronic adminstration of resveratrol or N-PEP-12 can restore vasorelaxation. This ability of resveratrol and $\mathrm{N}$ PEP-12 in aging rats might be related to their antioxidant properties [13,17,19]. Furthermore, chronic administration of resveratrol or N-PEP-12 appears not to modify the blood pressure and heart rate.

\section{Acknowledgements}

This study was supported by a VIEP-BUAP grant (No. FLAG-SAL11-G) and CONACYT grants (No. 129303 and 138663) to $G$ Flores. We also want to thank Dr. Carlos Escamilla for his help with the animal care. We are grateful to Ever NeuroPharma GmbH, Unterach, Austriafor the N-PEP-12 gift. RAVR and EM acknowledges the CONACYT for the scholarship. JFH, MJGV, GLL and GF acknowledge the National Research System of Mexico for membership. Thanks to KirenUbhi for editing the English language text.

\section{REFERENCES}

[1] M. Yadav, S. Jain, A. Bhardwaj, R. Nagpal, M. Puniya, R. Tomar, V. Singh, O. Parkash, G. B. Prasad, F. Marotta and H. Yadav, "Biological and Medicinal Properties of Grapes and Their Bioactive Constituents: An Update,” Journal of Medicinal Food, Vol. 12, No. 3, 2009, pp. 473-484. http://dx.doi.org/10.1089/jmf.2008.0096

[2] Z. Ungvari, G. Kaley, R. de Cabo, W. E. Sonntag and A. Csiszar, "Mechanisms of Vascular Aging: New Perspectives,” Journals of Gerontology Series A: Biological Sciences and Medical Sciences, Vol. 65, No. 10, 2010, pp. 1028-1041. http://dx.doi.org/10.1093/gerona/glq113

[3] A. Csiszar, Z. Ungvari, J. G. Edwards, P. Kaminski, M. S. Wolin, A. Koller and G. Kaley, “Aging-Induced Phenotypic Changes and Oxidative Stress Impair Coronary Arteriolar Function," Circulation Research, Vol. 90, No. 11, 2002, pp. 1159-1166. http://dx.doi.org/10.1161/01.RES.0000020401.61826.EA

[4] A. Csiszar, N. Labinskyy, Z. Orosz, Z. Xiangmin, R. Buffenstein and Z. Ungvari, "Vascular Aging in the LongestLiving Rodent, the Naked Mole-Rat,” American Journal of Physiology, Vol. 293, No. 2, 2007, pp. H919-H927.

[5] A. J. Donato, I. Eskurza, A. E. Silver, A. S. Levy, G. L. Pierce, P. E. Gates and D. R. Seals, "Direct Evidence of Endothelial Oxidative Stress with Aging in Humans: Relation to Impaired Endothelium Dependent Dilation and Upregulation of Nuclear Factor-KappaB,” Circulation Research, Vol. 100, No. 1, 2007, pp. 1659-1666. http://dx.doi.org/10.1161/01.RES.0000269183.13937.e8

[6] M. Frombaum, S. Le Clanche, D. Bonnefont-Rousselot and D. Borderie, "Antioxidant Effects of Resveratrol and Other Stilbene Derivatives on Oxidative Stress and NO Bioavailability: Potential Benefits to Cardiovascular Diseases,” Biochimie, Vol. 94, No. 2, 2012, pp. 269-276. http://dx.doi.org/10.1016/j.biochi.2011.11.001

[7] X. A. Alvarez, L. Corzo, M. Laredo, C. Sampedro, R.
Cacabelos, M. Windisch, H. Moessler and T. H. Crook, "Neuropeptide Dietary Supplement N-PEP-12 Enhances Cognitive Function and Activates Brain Bioelectrical Activity in Healthy Elderly Subjects," Methods and Findings in Experimental and Clinical Pharmacology, Vol. 27, No. 7, 2005, pp. 483-487. http://dx.doi.org/10.1358/mf.2005.27.7.914776

[8] T. H. Crook, S. H. Ferris, X. A. Alvarez, M. Laredo and H. Moessler, "Effects of N-PEP-12 on Memory among Older Adults," International Clinical Psychopharmacology, Vol. 20, No. 2, 2005, pp. 97-100.

http://dx.doi.org/10.1097/00004850-200503000-00006

[9] D. Volc, A. Alvarez and H. Moessler, “Cognitive Effects of the Novel Neuroprotective Dietary Supplement NPEP-12: Evidences from a Self-Assessment Study,” Jatros, Neurologie and Psychiatrie, 2005, pp. 1-7.

[10] F. Sanchez, M. de J. Gomez-Villalobos, I. Juárez, L. Quevedo and G. Flores, "Dendritic Morphology on Neurons from Prefrontal Cortex, Hippocampus and Nucleus Accumbens in Altered in Adult Spontaneously Hypertensive Rats,” Synapse, Vol. 65, No. 3, 2011, pp. 198-206. http://dx.doi.org/10.1002/syn.20837

[11] J. G. Lopez-Lopez, J. Moral-Sanz, G. Frazziano, M. J. Gomez-Villalobos, L. Moreno, C. Menendez, J. FloresHernandez, J. A. Lorente, A. Cogolludo and F. PerezVizcaino, “Type 1 Diabetes-Induced Hyper-Responsiveness to 5-Hydroxytryptamine in Rat Pulmonary Arteries via Oxidative Stress and Induction of Cyclooxygenase-2," Journal of Pharmacology and Experimental Therapeutics, Vol. 338, No. 1, 2011, pp. 400-407.

http://dx.doi.org/10.1124/jpet.111.179515

[12] A. G. Rajapakse, G. Yepuri, J. M. Carvas, S. Stein, C. M. Matter, I. Scerri, J. Ruffieux, J. P. Montani, X. F. Ming and Z. Yang, "Hyperactive S6K1 Mediates Oxidative Stress and Endothelial Dysfunction in Aging: Inhibition by Resveratrol,” PLoS One, Vol. 6, No. 4, 2011, e19237. http://dx.doi.org/10.1371/journal.pone.0019237

[13] E. V. Serkina, O. A. Gromova, I. Iu. Torshin, N. Iu. Sotnikova and A. A. Nikonov, "Cerebrolysin Alleviates Perinatal CNS Disorders through the Autoimmune Modulation and Antioxidant Protection," Zhurnal Nevrologii $i$ Psikhiatrii Imeni S.S. Korsakova, Vol. 108, No. 11, 2008, pp. 62-66.

[14] J. Behbahani, S. J. Thandapilly, X. L. Louis, Y. Huang, Z. Shao, M. A. Kopilas, P. Wojciechowski, T. Netticadan and H. D. Anderson, "Resveratrol and Small Artery Compliance and Remodeling in the Spontaneously Hypertensive Rat," American Journal of Hypertension, Vol. 23, No. 12, 2010, pp. 1273-1278. http://dx.doi.org/10.1038/ajh.2010.161

[15] A. M. El-Mowafy, M. Alkhalaf and N. N. Nassar, "Resveratrol Reverses ET-1-Evoked Mitogenic Effects in Human Coronary Arterial Cells by Activating the Kinase$\mathrm{G}$ to Inhibit ERK-Enzymes," International Journal of Cardiology, Vol. 136, No. 3, 2009, pp. 263-269. http://dx.doi.org/10.1016/j.ijcard.2008.04.094

[16] H. J. Ma, Y. K. Cao, Y. X. Liu, R. Wang and Y. M. Wu, "Microinjection of Resveratrol into Rostral Ventrolateral Medulla Decreases Sympathetic Vasomotor Tone through Nitric Oxide and Intracellular $\mathrm{Ca}^{2+}$ in Anesthetized Male 
Rats,” Acta Pharmacologica Sinica, Vol. 29, No. 6, 2008, pp. 906-912.

http://dx.doi.org/10.1111/j.1745-7254.2008.00827.x

[17] M. Subramanian, P. Balasubramanian, H. Garver, C. Northcott, H. Zhao, J. R. Haywood, G. D. Fink, S. M. Mohan Kumar and P. S. Mohan Kumar, "Chronic Estradiol-17 $\beta$ Exposure Increases Superoxide Production in the Rostral Ventrolateral Medulla and Causes Hypertension: Reversal by Resveratrol,” American Journal of Physiology. Regulatory, Integrative and Comparative Physiology, Vol. 300, No. 6, 2011, pp. R1560-1568. http://dx.doi.org/10.1152/ajpregu.00020.2011

[18] N. Toda and T. Okamura, "Modulation of Renal Blood Flow and Vascular Tone by Neuronal Nitric Oxide Synthase-Derived Nitric Oxide," Journal of Vascular Re- search, Vol. 48, No. 1, 2011, pp. 1-10. http://dx.doi.org/10.1159/000317395

[19] P. A. Kroon, A. Iyer, P. Chunduri, V. Chan and L. Brown, "The Cardiovascular Nutrapharmacology of Resveratrol: Pharmacokinetics, Molecular Mechanisms and Therapeutic Potential," Current Medicinal Chemistry, Vol. 17, No. 23, 2010, pp. 2442-2355. http://dx.doi.org/10.2174/092986710791556032

[20] J. W. Rush, J. Quadrilatero, A. S. Levy and R. J. Ford, "Chronic Resveratrol Enhances Endothelium-Dependent Relaxation but Does Not Alter eNOS Levels in Aorta of Spontaneously Hypertensive Rats,” Experimental Biology and Medicine (Maywood), Vol. 232, No. 6, 2007, pp. 814-822. 